Service social

\title{
Pauvreté urbaine et exclusion sociale. Les nouvelles figures du travail social auprès des personnes et des communautés locales en difficulté
}

\section{Louis Favreau et Lucie Fréchette}

Volume 44, numéro 3, 1995

Pauvreté

URI : https://id.erudit.org/iderudit/706707ar

DOI : https://doi.org/10.7202/706707ar

Aller au sommaire du numéro

Éditeur(s)

École de service social de l'Université Laval

ISSN

1708-1734 (numérique)

Découvrir la revue

Citer cet article

Favreau, L. \& Fréchette, L. (1995). Pauvreté urbaine et exclusion sociale. Les nouvelles figures du travail social auprès des personnes et des communautés locales en difficulté. Service social, 44(3), 71-93.

https://doi.org/10.7202/706707ar
Résumé de l'article

Le présent texte aborde trois questions: la première a trait à l'insuffisance des différentes analyses de la pauvreté et à sa relecture par l'introduction de la notion d'exclusion sociale. La seconde renvoie à l'émergence d'un double renouvellement des pratiques: le renouvellement de l'intervention psychosociale par une approche de prévention sociale et de promotion de réseaux et le renouvellement de l'intervention communautaire par une approche de développement local. Enfin, nous avançons la proposition suivante : depuis bientôt dix ans, l'émergence de corporations de développement communautaire ( $\mathrm{CDC}$ ) et de corporations de développement économique communautaire (CDEC) illustre ce double renouvellement des pratiques entant que nouveaux dispositifs d'accompagnement dans

l'empowerment des personnes et des communautés locales en difficulté. 


\section{Pauvreté urbaine et exclusion sociale Les nouvelles figures du travail social auprès des personnes et des communautés locales en difficulté}

Louis FAVREAU

Travailleur social, sociologue et professeur en travail social Université du Québec à Hull

LUCie FRÉCHETTE Psychologue et professeure en travail socia, Université du Québec à Hull

Le présent texte aborde trois questions: la première a trait à l'insuffisance des différentes analyses de la pauvreté et à sa relecture par l'introduction de la notion d'exclusion sociale. La seconde renvoie à l'émergence d'un double renouvellement des pratiques: le renouvellement de l'intervention psychosociale par une approche de prévention sociale et de promotion de réseaux et le renouvellement de l'intervention communautaire par une approche de développement local. Enfin, nous avançons la proposition suivante: depuis bientôt dix ans, l'émergence de corporations de développement communautaire $(C D C)$ et de corporations de développement économique communautaire (CDEC) illustre ce double renouvellement des pratiques 
en tant que nouveaux dispositifs d'accompagnement dans l'empowerment des personnes et des communautés locales en difficulté1.

\section{LES LIMITES DES RECHERCHES ET DES THÉORIES SUR LA PAUVRETÉ}

La pauvreté urbaine dans le Québec d'aujourd'hui n'exige pas une longue démonstration. Sa description a maintes et maintes fois été faite par des institutions publiques ou des organismes du milieu. $C^{\prime}$ est la lecture épidémiologique qui prévaut ${ }^{2}$.

La lacune majeure de la lecture épidémiologique demeure le fait de caractériser la pauvreté en s'en tenant à la description de l'existence de groupes socio-économiquement faibles (sur le plan de la santé, de l'alimentation, de l'éducation, du revenu...), groupes qui deviennent par la suite des "populations cibles», des "groupes à risque " à qui il faut accorder la priorité dans l'intervention. Cela donne à cette pauvreté le caractère très net d'une "construction institutionnelle » (Lesemann, 1993: 589) ${ }^{3}$. L'inconvénient de cette lecture, c'est qu'elle ne correspond pratiquement jamais à des situations concrètes, $c^{\prime}$ est-à-dire à des communautés et à des régions précises vivant des processus sociaux déstructurants. Rarement, surtout en ce qui a trait à la pauvreté urbaine, s'attarde-t-on à la dimension territoriale de celle-ci, à sa concentration de plus en plus marquée, à la spécialisation sociale de l'espace urbain. Les rapports du Conseil des affaires sociales du Québec (CAS, 1989 et 1990) sont $d^{\prime}$ heureuses exceptions. Rarement aussi s'attarde-t-on à intégrer les deux dimensions de la pauvreté, sa dimension plus sociale faite de vulnérabilité de personnes, de groupes sociaux et de communautés et sa dimension économique avec le déclin des économies locales... Finalement, rarement aussi s'attarde-t-on à examiner le travail $d^{\prime}$ organisation communautaire qui s'y accomplit et l'état des forces vives de ces milieux en difficulté.

Une revue sommaire des écrits portant sur la pauvreté fait globalement ressortir trois types d'explications auxquelles se greffent des modes $d^{\prime}$ intervention correspondants. Sur un premier registre d'explications, on fait surtout ressortir les facteurs sociosanitaires ou psychosociaux associés à la pauvreté. Sur le plan psychosocial, les gens pauvres expérimentent alors la pauvreté ou ses conséquences comme des pathologies, des manques. Selon cette vision, les personnes pauvres ne réussissent pas à s'adapter à la modernisation de la société (analphabétisme, absence de sens de l'organisation, 
mésadaptation à la vie urbaine...). On y retrouve aussi l'idée que la pauvreté est accidentelle, conséquence d'événements de la vie tels une séparation ou un divorce, la grossesse de femmes seules, l'entrée en chômage de travailleurs de plus de 45 ans à la suite d'une fermeture d'entreprise, des immigrants trouvant refuge au Canada (réfugiés politiques ou économiques). Cette conception de la pauvreté conduit à privilégier une intervention où priment les mesures d'urgence: le dépannage, la relation d'aide auprès des personnes ou des familles en situation de crise, la campagne publique de sensibilisation (contre la violence ou contre les abus du tabac...) accompagnent généralement ce type de diagnostic de la pauvreté.

Un second registre d'explications met d'abord en relief les facteurs socioculturels. La pauvreté est perçue comme un phénomène associé à un mode de vie. Souvent héritée, cette pauvreté en arrive à développer chez ceux qui la vivent une culture de dépendance que l'assistance sociale, qu'elle soit publique ou privée, ne fait qu'entretenir. À l'inverse de cette dernière, une troisième explication met l'accent sur les facteurs socio-économiques associés à la pauvreté. La pauvreté est alors examinée sur une base plus structurelle, c'est-à-dire comme pauvreté de "système", celle d'une économie de marché laissée à elle-même et provoquant de graves iniquités sur le plan social.

Dans ces deux derniers cas, on fait généralement appel à l'État pour travailler à lutter contre cette pauvreté, mais dans deux directions opposées: celle du workfare et celle du welfare. La première, en identifiant la culture de la pauvreté comme variable lourde, veut exiger des "pauvres " qu' « ils fassent leur part " parce que l'assistance sociale cultive trop la dépendance. C'est l'inflexion néolibérale. La seconde, identifiant l'économie de marché comme clé d'explication, veut plutôt que l'État "fasse beaucoup", en l'exigeant par la mobilisation collective et des actions de défense de droits sociaux. C'est la thèse traditionnelle des mouvements sociaux qui rejoint sur le plan politique le projet social-démocrate, $c^{\prime}$ est la thèse du renforcement de l'État-providence, première génération (1960-1975), thèse défendue par ceux qui préconisent une intervention vigoureuse de l'État en matière de transferts sociaux. Or, deux choses ont profondément changé : I'État-providence est en crise ${ }^{4}$ et la pauvreté s'est muée en exclusion.

Mais la pauvreté d'aujourd'hui, celle des années 90, est-elle la même que la pauvreté d'hier, celle des années 60 ?

À première vue banale, cette question nous force cependant à modifier la perspective, à renouveler nos approches. Si effectivement 
la pauvreté d'aujourd'hui n'est plus la même, cela nous amène à devoir réviser significativement nos pratiques. La pauvreté d'aujour$d^{\prime}$ hui est effectivement en train de changer de nature. Pourquoi? $C^{\prime}$ est qu'elle est en voie de devenir de plus en plus une pauvreté d'exclusion liée directement au problème de l'emploi pour des couches de plus en plus nombreuses de la population. Moins une pauvreté héritée chez des groupes bien spécifiques, elle pourrait plutôt se caractériser comme étant une pauvreté de crise de secteurs de plus en plus importants de la population, secteurs pour qui le travail représente un profil "normal», un horizon effectif, parce que les personnes concernées disposent d'une certaine compétence, $d^{\prime}$ une culture du travail ou d'une scolarité de base significative.

\section{REGARD SUR LA PAUVRETÉ D'AUJOURD'HUI À TRAVERS LE PRISME DE CHANGEMENTS SOCIAUX RÉCENTS}

La crise de l'emploi, la crise des quartiers et la spécialisation sociale de l'espace urbain font aujourd'hui plus qu'hier partie des tendances fortes du paysage social. Le visage traditionnel de la pauvreté s'en trouve largement modifié. Examinons de plus près cette avancée.

Des années 60 aux années 90, un grand nombre de changements sont venus modifier la dynamique sociale et économique des communautés locales. La structure du chômage a changé : il y a désormais beaucoup plus de chômage de longue durée. Le portrait des prestataires de l'aide sociale a aussi changé : ceux-ci sont nettement plus jeunes et majoritairement aptes au travail. Chômage prolongé et dépendance de la sécurité du revenu sont des éléments qui affectent l'identité personnelle et la qualité de la vie familiale. Le réseau social risque le rétrécissement et la menace de la pauvreté peut faire sourdre peu à peu le sentiment de honte (Gaulejac, 1989). De plus, il est connu que l'isolement social et la pauvreté dans un milieu créent des conditions favorables à l'émergence de comportements de violence familiale et $d^{\prime}$ abus physique envers les enfants (Bouchard, 1991).

Le portrait des immigrants intégrant les quartiers a aussi changé. L'immigration est aujourd'hui une immigration surtout en provenance du Sud qui se compose souvent de familles entières de réfugiés politiques ou économiques. L'immigration de main-d'œuvre spécialisée venant de l'Europe $n^{\prime}$ est plus chose courante comme nous I'avions connue. La tendance à l'appauvrissement modifie par ailleurs les statuts sociaux, car il y a affaiblissement sinon effacement de la mobilité sociale pour les nouvelles générations. 


\section{La spécialisation sociale de l'espace urbain}

De plus, il faut enregistrer la tendance de la pauvreté urbaine et du chômage à se concentrer et à épouser le découpage social du territoire des villes. L'espace social urbain devient alors de plus en plus inégalitaire, soit d'un côté les pauvres dans les centres-villes et de l'autre les familles de classe moyenne à la périphérie ou en banlieue (Favreau et Hurtubise,1993; Laferrière, 1992; CAS, 1989). Un mémoire élaboré par les maires des six principales villes-centres du Québec résume bien la situation. Il existe en effet:

...Une polarisation sociale, démographique ou culturelle croissante entre le centre et la périphérie. D'une part, la villecentre concentre, dans ses quartiers, les populations les moins mobiles, les plus défavorisées et les plus dépendantes des services publics et des programmes de soutien du revenu, et qui n'ont, par voie de conséquence, pas accès à la propriété résidentielle. D'autre part, les banlieues regroupent une population en général plus homogène, plus favorisée au plan socio-économique et moins dépendante des services publics... (Les villes-centres... 1993: 9)

La pauvreté dans ce contexte de spécialisation de l'espace urbain est un état de fait qui affecte non seulement les adultes, mais la famille entière et de façon particulière les enfants. En effet, la pauvreté n'est plus tellement le lot des personnes âgées, mais bien celui des jeunes et des enfants. Au Québec, près de $20 \%$ des familles sont pauvres, ce qui touche directement 322000 enfants (les moins de 18 ans). Ces derniers sont les grands perdants d'une société qui devrait plutôt les initier à une citoyenneté stimulante.

\section{L'emploi au cour du problème de la pauvreté}

On s'en rend beaucoup plus compte aujourd'hui qu'hier, l'emploi organise les clivages et les dynamismes sociaux dans les centres urbains comme dans les petites villes et les communautés rurales, dans les régions centrales comme dans les régions périphériques. En effet, aujourd'hui, au sein de la population active au Québec, il y a trois fois plus de sans-emploi qu'au début des années 60 , moment d'expansion de l'économie et de l'État-providence. Et les sans-emploi demeurent deux à trois fois plus longtemps "sur » le chômage qu'auparavant (Tremblay, 1990: 31-66). Le degré d'exposition au risque du chômage s'est aussi considérablement élevé pour de plus en plus de familles, car le travail se précarise.

La question de I'emploi est donc devenue envahissante. Depuis dix ans, une bonne partie des débats sur la jeunesse, sur l'immigration, 
sur la pauvreté, sur les quartiers, de même que bon nombre de questions posées aux intervenants sociaux dans les CLSC, dans les organisations syndicales, les groupes de femmes ou les organisations communautaires, nous renvoient au problème de l'emploi. Ce qui relance, du coup, le débat sur la qualité de ces emplois, sur leur durée... Pourquoi cette obsession? Précisément parce qu'aujourd'hui avoir ou ne pas avoir d'emploi demeure le premier et principal vecteur de la qualification sociale ou de la disqualification sociale (Paugam, 1991), c'est-à-dire de la qualité de vie, de la reconnaissance sociale par le travail et de l'organisation d'un mode de vie relié à ce travail. Ou son contraire! La situation de l'emploi au Québec ne ferait pas que favoriser une augmentation des inégalités sociales. Elle serait en voie d'introduire une insidieuse fracture sociale entre les « in» et les « out» (Roman, 1993).

\section{Dualisme social et géographique : les risques $d^{\prime} u n e$ fracture sociale}

La pauvreté d'aujourd'hui vue sous l'angle du couple chômageemploi frappe très inégalement: elle frappe d'abord les femmes plus que les hommes, elle frappe les jeunes, elle frappe les moins scolarisés. Elle affecte les familles à parent unique dont la responsabilité, on le sait, est assumée en majorité par des femmes. Elle touche aussi les jeunes familles et donc les enfants en bas âge, de même que les travailleurs plus âgés (45 ans et plus) et leur famille.

La pauvreté affecte durement la qualité du tissu social des communautés locales. En fait, nous nous orientons vers une société où les tensions liées à l'exclusion prennent autant d'importance et peut-être plus que celles liées aux milieux de travail. En effet, d'une part il est possible de constater que le travail précaire augmente plus vite que le travail permanent, que la coupure emploi/logement prend plus d'ampleur et que l'immigration nouvelle provoque un plus grand choc des cultures. L'exclusion devient alors une pente douce vers un faible sentiment $d$ 'appartenance sociale et finalement une citoyenneté de seconde zone ou passive de plus en plus concentrée dans des communautés locales en déclin.

D'autre part, depuis au moins trois décennies, de nombreux dispositifs ont été mis en place dans les entreprises à la faveur de la montée de la "société salariale et providentielle", telles des conventions collectives et des mesures de transfert social de l'État (régimes de retraite, régimes d'assurance-salaire...). II y a donc régulation possible des conflits par l'introduction de dispositifs $d^{\prime}$ " intégration 
sociale" même si cette intégration s'opère souvent sous le mode conflictuel (par des affrontements telles des grèves, des manifestations...).

Mais là où l'exclusion tend à l'emporter, les dispositifs d'amélioration des conditions de vie et donc $d^{\prime}$ " intégration» sont plus faibles pour les groupes sociaux les plus touchés - femmes, jeunes, immigrants et travailleurs de 45 ans et plus - et pour les communautés en difficulté. Le dualisme social et géographique risque alors de se transformer en fracture sociale provoquant une rupture de lien plutôt qu'une "intégration conflictuelle». Les travailleurs sociaux communautaires et les intervenants sociaux en général sont donc très fortement interpellés par cette nouvelle situation qui commande une révision des modes traditionnels d'intervention.

\section{PAUVRETÉ ET DÉCLIN DES COMMUNAUTÉS LOCALES: ESQUISSE D'UN CYCLE NÉGATIF DE CHANGEMENT SOCIAL}

Le nouveau visage de la pauvreté ne peut plus être occulté. La pauvreté urbaine, par delà les statistiques rendant compte du faible revenu des familles, se comprend et s'explique d'abord par un cycle négatif de changement social lié aux interventions déstructurantes des entreprises et des institutions au plan local. La pauvreté urbaine est en premier lieu affaire de quartier où des facteurs macroéconomiques se conjuguent avec des facteurs plus spécifiquement locaux pour structurer ou déstructurer des milieux.

Dans le cadre de recherches effectuées ces dernières années dans plusieurs régions du Québec (notamment dans I'Outaouais), nous avons pu effectuer une série d'entretiens, de visites de quartier et le repérage de projets d'intervention novateurs. Nos observations en rapport avec l'émergence des nouvelles formes de pauvreté et leur encastrement dans des quartiers nous ont amenés à esquisser les principaux fils directeurs de ce que nous appelons le cycle négatif de changement social des communautés locales. Ces études ont été menées dans des quartiers en difficulté de plusieurs villes. Ce cycle négatif se caractérise par six variables de base - dont certaines sont de niveau macro-social et d'autres plus micro-social - illustrées au tableau 1 de la page suivante.

Une bonne synthèse de ce cycle a été illustrée dans les propos tenus en entrevue par un leader communautaire de longue date:

Ici ça vieillit. La commission scolaire a fermé une école parce qu'il n'y avait pas suffisamment d'enfants... Aussi, il y a des 


\section{Tableau 1}

\section{Cycle négatif de changement social}

Familles

Écoles

Économie locale

\section{Politique}

de la municipalité

Économie

du centre-ville
Des divorces et des séparations : femmes candidates à l'aide sociale; pères de famille de 45 ans et plus mis à pied ; pourcentage élevé de familles monoparentales et de jeunes couples avec enfants en situation économique difficile.

Un fort pourcentage de jeunes en situation de décrochage, la fermeture d'écoles de quartier...

La fermeture de petits commerces (quincaillerie, épicerie...) dans les quartiers.

Le déménagement de succursales bancaires vers le centre-ville.

Un développement prioritaire du centre-ville par le secteur privé ("gentrification »...).

Un espace résidentiel grugé au bénéfice de la grande entreprise (privée ou publique).

Une augmentation de l'insécurité au sein des populations locales par l'arrivée d'un nouveau type de commerces (bars...) et une augmentation de la criminalité, des « gangs »...

Une politique de désengagement (de laissez-faire) : le déménagement de services de caractère public (bureau de poste...) dans d'autres secteurs de la ville...

commerces qui s'en vont. Et puis, les gens sont obligés d'aller à l'extérieur du quartier pour obtenir des services qu'ils avaient avant dans leur quartier. Par exemple, il y avait une banque sur la rue Saint-Louis. Elle a déménagé au centreville... Les épiceries, il y en a de moins en moins. C'est drainé vers les gros (Loblaws...). Conséquence, les gens ont moins de fierté, leurs rues deviennent délabrées... les jeunes familles cherchent à partir... Les jeunes, quand ils grandissent, quand vient le temps de se marier, s'en vont à l'extérieur...

En outre, la pauvreté sur laquelle nous avons mené enquête s'est révélée moins homogène que nous l'avions prévu. Les visites de quartier et les enquêtes-terrain que nous avons menées ont en effet 
mis en relief la complexité de cette pauvreté urbaine, ce qui nous conduit à proposer ici un profil contrasté de la pauvreté à partir d'une typologie de ces quartiers dits de pauvreté autour de cinq indicateurs présentés au tableau 2.

\section{TABleaU 2}

Typologie des quartiers pauvres en milieu urbain

\begin{tabular}{|c|c|c|c|}
\hline $\begin{array}{l}\text { Type de quartier/ } \\
\text { dimensions }\end{array}$ & $\begin{array}{c}\text { Quartiers de } \\
\text { grande pauvreté } \\
\text { (en crise) }\end{array}$ & $\begin{array}{c}\text { Quartiers } \\
\text { en difficulté }\end{array}$ & $\begin{array}{c}\text { Quartiers en voie } \\
\text { d'appauvrissement } \\
\text { ou d'exclusion... }\end{array}$ \\
\hline $\begin{array}{l}\text { Situation socio- } \\
\text { économique } \\
\text { de la population }\end{array}$ & $\begin{array}{l}\text { \% élevé de familles } \\
\text { à parent unique, de } \\
\text { personnes âgées, } \\
\text { d'assistés sociaux, } \\
\text { de sans-emploi ; } \\
\text { prédominance du } \\
\text { logement social... }\end{array}$ & $\begin{array}{l}\text { \% élevé de jeunes } \\
\text { en chômage, de } \\
\text { décrochage scolaire, } \\
\text { de jeunes familles à } \\
\text { faible revenu... }\end{array}$ & $\begin{array}{l}\text { Situation socio- } \\
\text { économique se } \\
\text { détériorant un peu } \\
\text { plus d'année en } \\
\text { année }\end{array}$ \\
\hline $\begin{array}{l}\text { Situation de } \\
\text { l'économie locale }\end{array}$ & $\begin{array}{l}\text { Absence } \\
\text { d'économie locale, } \\
\text { existence d'une } \\
\text { économie } \\
\text { informelle }\end{array}$ & $\begin{array}{l}\text { Économie locale } \\
\text { faible }\end{array}$ & $\begin{array}{l}\text { Économie locale } \\
\text { disposant d'un } \\
\text { certain potentiel }\end{array}$ \\
\hline $\begin{array}{l}\text { Degré de dévelop- } \\
\text { pement de l'infra- } \\
\text { structure de } \\
\text { services de base }\end{array}$ & $\begin{array}{l}\text { Faiblesse marquée } \\
\text { des services de base } \\
\text { (accès transport } \\
\text { local, centre } \\
\text { commercial, } \\
\text { garderie...) }\end{array}$ & $\begin{array}{l}\text { Faiblesse de certains } \\
\text { services de base }\end{array}$ & $\begin{array}{l}\text { Services en voie de } \\
\text { détérioration }\end{array}$ \\
\hline $\begin{array}{l}\text { Force du tissu social } \\
\text { (cohésion ou } \\
\text { appartenance } \\
\text { sociale) }\end{array}$ & $\begin{array}{l}\text { Tissu social } \\
\text { faible, morcelé } \\
\text { (délinquance, } \\
\text { vandalisme...) }\end{array}$ & Tissu social affaibli & $\begin{array}{l}\text { Tissu social } \\
\text { potentiel assez fort }\end{array}$ \\
\hline $\begin{array}{l}\text { Vitalité communau- } \\
\text { taire (organismes } \\
\text { de type commu- } \\
\text { nautaire) }\end{array}$ & $\begin{array}{l}\text { Faible, nécessitant } \\
\text { une intervention } \\
\text { extérieure forte }\end{array}$ & $\begin{array}{l}\text { Organisation } \\
\text { communautaire } \\
\text { dans certains } \\
\text { champs } \\
\text { d'intervention }\end{array}$ & $\begin{array}{l}\text { Un certain } \\
\text { individualisme, } \\
\text { capacité de } \\
\text { mobilisation forte } \\
\text { autour d'enjeux } \\
\text { concrets }\end{array}$ \\
\hline
\end{tabular}

Loin d'être académique, cette distinction entre quartiers de grande pauvreté, quartiers en difficulté et quartiers en voie d'appauvrissement permet de cerner de façon plus précise les problèmes sociaux vécus par les familles de milieu défavorisé. En outre, elle 
permet de faire valoir la nécessité de types différents d'intervention. Par exemple, à une extrémité, les quartiers caractérisés par une grande pauvreté nécessiteront un investissement de recomposition du tissu social plus considérable et de longue durée. À l'autre pôle, les quartiers en voie d'appauvrissement invitent davantage à un travail de caractère préventif combinant tout à la fois $\mathrm{l}^{\prime}$ "économique» et le «social».

\section{LES NOUVELLES PAUVRETÉS APPELLENT AU RENOUVELLEMENT DES INTERVENTIONS SOCIALES.}

\section{Le renouvellement de la pratique passe par des approches préventives, de promotion de réseaux et de développement économique communautaire.}

L'appauvrissement des communautés locales constitue la toile de fond du renouvellement actuel des modes d'intervention et de l'émergence de stratégies innovatrices. Passer d'une intervention réactive qui pallie les urgences à une intervention proactive du développement local et de la prévention sociale constitue un impératif dans le contexte social actuel. De plus en plus d'intervenants diagnostiquent que si le cycle de changement social négatif peut être inversé, $c^{\prime}$ est bien par une intervention jouant sur plusieurs claviers, c'est-à-dire s'attaquant simultanément à plusieurs problèmes et intervenant sur deux plans, celui de la prévention sociale et celui du développement local. En ce sens, Claude Julien résume avec justesse le constat initial sous-jacent à ce diagnostic :

Dans les quartiers réputés difficiles, les travailleurs sociaux connaissent d'expérience la parfaite vanité de toute intervention sectorielle, de toute action, si bien intentionnée soit-elle, qui se limiterait à une seule cause de la "fracture sociale». L'efficacité requiert d'agir simultanément sur tous les fronts: scolarisation proprement dite, activités culturelles et de loisirs, conditions de logement et de santé, etc. (Julien, 1995 :17)

En effet, de ce que nous avons pu observer dans de nouvelles pratiques, notamment dans celles des corporations de développement communautaire $(C D C)$ et des corporations de développement économique communautaire (CDEC), la prévention sociale favorise globalement trois choses: 1 ) elle travaille à contrer la rupture des liens familiaux; 2) elle contribue à la réduction de l'isolement des personnes et freine l'affaiblissement des liens sociaux; 3) elle stimule l'accroissement de la compétence des personnes et des groupes. 
De son côté, le développement local favorise: 1) la réinsertion sociale des prestataires de la sécurité du revenu et des chômeurs de longue durée, de même que la lutte contre la précarité de l'emploi, notamment chez les jeunes; 2) la formation qualifiante des populations résidantes; 3) la reconquête du contrôle des communautés sur leur développement. ... Déplions ici l'ensemble de cette proposition.

La prévention, dans une perspective qui n'est plus seulement sociosanitaire mais bien sociale, induit, dans sa vision élargie, la promotion de réseaux d'entraide. Elle s'avère une passerelle pertinente entre la relation d'aide et l'organisation des communautés (Guay, 1984; Sanicola 1994). On perçoit d'ailleurs une ouverture dans cette direction, par exemple dans les plus récents énoncés de politiques en matière de santé mentale du Gouvernement du Québec (1992). Le Comité de la santé mentale du Québec a voulu pousser plus loin la réflexion en confiant à un groupe de travail l'étude des liens entre la pauvreté et la santé mentale (Robichaud et al., 1994).

L'approche préventive appelle des pratiques plus larges qui prennent davantage en considération l'ensemble de la personne et des réseaux d'une communauté donnée. Car cette approche s'oriente vers l'élaboration de solutions globales, prend la forme de pratiques rejoignant un plus grand nombre de personnes, par plusieurs points d'attaque (Chamberland et al., 1993). Comme le signalent pertinemment les travaux du Comité de la santé mentale du Québec, I'articulation bien planifiée de plusieurs stratégies et méthodes d'intervention peut mieux favoriser l'atteinte d'objectifs sociaux (Blanchet et al., 1993)

La prévention sociale comme le développement local sont d'abord des approches et non des méthodes spécifiques d'intervention. Elles agissent à la manière $d^{\prime}$ un canevas qui donne une direction à l'analyse des problèmes sociaux et à l'élaboration de stratégies $d^{\prime}$ intervention où la relation $d^{\prime}$ aide devient développement des personnes, mais aussi développement des groupes et des communautés. La prévention sociale élargit le cadre d'intervention des travailleurs du social en rappelant aux tenants de l'intervention auprès des personnes que leur pratique de soutien ou d'aide psycho-sociale n'a de sens que si elle est complétée par des actions collectives pour I'amélioration des conditions de vie. Elle rappelle aux tenants de I'organisation communautaire qu'ils ne peuvent faire l'économie d'une mobilisation qui part des centres d'intérêt spécifiques des personnes et des groupes et de leur représentation particulière de la santé et du bien-être.

Les CDEC et les CDC combinent bien ces deux approches. D'abord parce qu'elles interviennent sur une base territorialisée 
plutôt qu'exclusivement en fonction de populations cibles. Un certain nombre de CLSC vont de plus en plus dans le même sens en mettant en œuvre une approche-milieu par le travail d'équipes multidisciplinaires organisées sur la base de quartiers plutôt que sur la base d'étapes de vie (enfance-famille, etc.). Ensuite les CDEC et les CDC font porter l'effort simultanément sur plusieurs dimensions de la pauvreté au sein de communautés locales: la famille, l'école, l'emploi et les entreprises locales, la formation de la population résidante, la revitalisation du centre-ville en collaboration avec la municipalité et l'utilisation maximale des programmes publics $d^{\prime}$ 'insertion et de formation de la main-d'œuvre. Prévention sociale et développement local se rejoignent donc ici dans leurs dimensions complémentaires pour contrer la pauvreté à partir de stratégies diversifiées. L'intervention est composée d'activités opérant simultanément sur plusieurs registres. C'est ce que tente d'illustrer le tableau 3.

Dans de nombreuses communautés locales, il existe déjà des initiatives de prévention sociale dans les écoles ou avec les familles en difficulté comme des groupes d'entraide socio-économique de type cuisines collectives, des initiatives d'insertion par l'économique avec des jeunes comme les Coopératives jeunesse de services (CJS) qui aident, notamment, à contrer le décrochage scolaire. II existe déjà des initiatives de développement communautaire, tels des organismes communautaires d' "employabilité", des écoles-entreprises, des entreprises coopératives et communautaires, des entreprises $d^{\prime}$ insertion sociale ${ }^{5}$, bref, pour employer l'expression de Jean-Louis Laville, des "services de proximité " (Laville, 1992).

Cet ensemble d'initiatives qui sont surtout apparues dans les dix dernières années prennent de l'ampleur et sont de plus en plus reconnues. Elles font toutefois face à des défis importants: celui de la consolidation d'acquis sans cesse fragilisés par le contexte d'un soutien public précaire et par l'absence ou la faiblesse de structures d'appui proches d'elles. Le renforcement du pouvoir d'action de ces initiatives sur la dynamique socio-économique locale et régionale $\mathrm{s}^{\prime}$ est imposé et a fait émerger des CDEC et des CDC .

Précisons ici qu'un certain nombre de groupes se sont donné des regroupements sur le plan sectoriel. En additionnant leurs forces, ils ont pu donner de l'ampleur aux causes défendues et accréditer la place du "communautaire». Mais avec la modification du visage de la pauvreté, le nouveau défi posé est devenu celui d'une intervention inscrite dans une logique territoriale. Autès (1992) a raison de distinguer deux logiques sociales (plutôt qu'une) dans la lutte contre la pauvreté: la logique catégorielle et la logique territoriale. Cette 


\section{TABleAU 3}

\section{Intervention multiactivités contrant la pauvreté}

\section{Dimensions \\ de l'intervention}

Familles

Écoles

Économie locale

Économie du centre-ville et de la ville

Politique de

la municipalité

Politique des pouvoirs publics centraux dans les communautés locales

\section{Cycle positif de changement introduit par une intervention multiactivités}

Stimulation de la création de réseaux sociaux et économiques : cuisines collectives, maisons de quartier, halte-garderie...

Stimulation de la relation positive école-travail par des coopératives jeunesse de services, des maisons de jeunes...

Soutien au redéveloppement de l'économie de proximité : micro-entreprises (biens et services) ; maillage entre ces entreprises...

Achat et gestion d'espaces aménageables en fonction des populations locales et du développement du centre-ville simultanément : fonds de fiducie foncière communautaire, coops $d^{\prime}$ habitation, petits centres commerciaux...

Politique équilibrée $d^{\prime}$ investissement et de soutien au développement de la ville et de ses quartiers en difficulté.

Politique active de développement des quartiers en difficulté : programmes de soutien à la formation de la main-d'œuvre résidante, aux entreprises locales en difficulté...

dernière nécessite aussi que soient construits des dispositifs spécifiques, lesquels sont orientés vers le développement d'ensemble des quartiers ou des communautés locales. Nombre d'intervenants sociaux concluaient lors d'entretiens qu'il était impératif de sortir les multiples projets du simple bricolage et de doter leur communauté de structures adéquates d'accompagnement de ces projets. Car les initiatives qu'ils ont aidé à mettre sur pied courent aujourd'hui plus qu'hier le risque de s'essouffler et d'avoir une faible continuité. Elles ont besoin d'environnements favorables et de leviers de développement. 


\section{Deux avenues prometteuses en travail social auprès des personnes et des communautés :}

Les CDC (Corporations de développement communautaire) et les CDEC (Corporations de développement économique communautaire)

À l'examen de nombreux projets, nous nous sommes rendu compte que là où existent des CDC (expérience en cours dans près d'une vingtaine de sous-régions [MRC] du Québec) et des CDEC (expérience en cours dans l'ensemble des arrondissements de la ville de Montréal et dans les quartiers centraux de la ville de Québec), les communautés parviennent beaucoup mieux, par un travail simultané sur plusieurs problèmes, à réintroduire un cycle positif de changement social.

Si I'on prend, à titre d'illustration, l'approche de développement économique communautaire telle que construite par les CDEC montréalaises, on constate que ces dernières peuvent par leurs mandats, leur structuration et leur association avec des partenaires divers réussir là où d'autres types d'initiatives $n^{\prime}$ ont pas été capables de faire émerger des projets disposant d'effets d'entraînement durables. Les CDEC, ces organismes sans but lucratif de type partenarial, favorisent en effet la coordination, la concertation et la solidarité des différents acteurs locaux des communautés concernées (CRÉECQ, 1993).

Mais comment ces nouveaux dispositifs d'intervention fonctionnent-ils pour concrétiser ces avantages? En premier lieu, les CDEC ont une mission de revitalisation économique et sociale des communautés en difficulté qu'elles desservent. Pour ce faire, elles ont adopté une intervention autour de trois axes:

a) La prévention par le développement des compétences des populations résidantes. À cet effet diverses mesures ont été privilégiées, telles que services de référence, de suivi, d'aide à la recherche d'emploi, activités de formation (visant l'augmentation du niveau de scolarité ou l'apprentissage d'un métier) pour des personnes exclues du marché du travail, etc. Par exemple RÉSO, une CDEC dans le sudouest de Montréal, a créé un centre d'aide aux sans-emploi (CASE) qui en 1994-1995 a fait de l'accompagnement auprès de 800 personnes.

b) Le développement local par l'intervention active sur le bassin d'emplois en améliorant la situation des entreprises locales: soutien à la gestion et au financement des entreprises existantes, appui au démarrage de nouvelles entreprises à partir d'un fonds communautaire de développement, promotion de l'embauche locale, etc. Par exemple, en 1994-1995 au RÉSO, 91 employeurs du sud-ouest ont fait appel à la CDEC pour combler un ou des postes de travail. 
c) La prévention et le développement local par le soutien à la recomposition du tissu social: face au décrochage scolaire, pour contrer I'isolement des familles prestataires de l'aide sociale... Par exemple, le RÉSO a soutenu le démarrage de Coopératives jeunesse de services, d'entreprises d'insertion sociale (Formétal) et d'entreprises communautaires (les Cuisines Jeanne-Leber). En outre, des initiatives de mise en réseau de cuisines collectives, de création de clubs de devoirs pour des enfants en difficulté, etc., ont surgi.

Les CDEC ont une démarche inédite en se constituant sur la base d'un partenariat local du secteur associatif ou communautaire (organisations communautaires et coopératives, groupes de femmes et syndicats), du secteur privé (entreprises et institutions financières) et du secteur public (municipalités et gouvernements centraux) pour favoriser les concertations et coordinations nécessaires à la revitalisation de ces quartiers. Si le partenariat réussit à susciter des participations croisées multiples où chacun y retrouve une partie de ses intérêts - ce qui n'est pas toujours le cas -, des synergies se créent entre les différents acteurs qui parviennent assez souvent à s'entendre autour de priorités de développement pour leur quartier.

Autre caractéristique de cette démarche de DEC, I'angle d'attaque des problèmes $n^{\prime}$ est pas uniquement social. L'intervention est tout à la fois "économique " et "sociale", c'est-à-dire qu'elle vise à maximiser la circulation de l'argent et la création d'emplois par la production et l'échange de biens et de services dans les quartiers euxmêmes. Bref, le quartier travaille à se recomposer comme premier marché des activités économiques tout en cultivant chez ses résidants le sentiment d'appartenance:

En quelque sorte la solidarité entre les habitants n'est plus cantonnée à la sphère des transferts sociaux (impôts, aides)... elle joue de manière localisée, par le biais de circuits courts de financement en étant directement en prise sur la sphère de la production. C'est là, à notre avis, l'innovation majeure de ce type de stratégie (Jacquier, 1993: 136).

Bref, à la différence des interventions classiques sur la pauvreté qui ne considèrent que la dimension sociale, cette double approche de prévention sociale et de développement économique communautaire fournit, à partir de ces nouveaux dispositifs que sont les CDC et les CDEC, les principaux ingrédients d'une stratégie intégrée et territorialisée de revitalisation sociale et économique en direction des personnes et des communautés en difficulté.

Les nouvelles avenues d'intervention offertes par les CDC et les CDEC permettent en effet de réunir sous un même toit des intervenants 
orientés tantôt vers le développement local par le soutien au développement d'entreprises et la formation qualifiante de la main-d'œuvre locale, tantôt vers la prévention par la mise en œuvre de stratégies d'insertion sociale de personnes prestataires de la sécurité du revenu ou de l'assurance-chômage, tantôt vers la recomposition du tissu social par l'entraide socio-économique. C'est que les CDC et les CDEC additionnent les initiatives en établissant des relais productifs entre elles. Les artisans de la prévention sociale et du développement local peuvent établir des passerelles entre les différents volets de travail portés par ces instances d'accompagnement des initiatives communautaires.

\section{Portée et liMites de CES INTERVENTIONS DE PRÉVENTION SOCIALE ET DE DÉVELOPPEMENT LOCAL}

\section{Des initiatives encore jeunes}

C'est grâce au travail de dirigeants d'organisations communautaires de quartier et d'intervenants sociaux, d'organisateurs communautaires de CLSC, d'animateurs de groupes de jeunes, de militants chrétiens socialement engagés, d'intervenantes de groupes de femmes qu'ont émergé les premières CDC et CDEC (1984-1985) dans les milieux populaires urbains en région et dans les grands centres. Des dirigeants de syndicats locaux et leur appareil régional (CSN et FTQ) et des dirigeants de certaines caisses populaires ou d'économie sont venus $s^{\prime} y$ greffer par la suite.

Ces initiatives sont encore au stade expérimental: elles ne sont encouragées ou soutenues par les pouvoirs publics que dans certaines régions ou certaines villes. Elles sont également fragiles, parce que soumises aux règles du jeu d'un financement souvent aléatoire des pouvoirs publics. Enfin, ces initiatives sont inégalement acceptées et reconnues au sein même du mouvement communautaire, syndical et coopératif. Mais elles représentent une tendance d'avenir:

En un temps où la crise de l'emploi accompagne la crise des grandes organisations, l'emploi se crée dans les petites entreprises comme dans le tissu associatif (Maire, 1987: 120).

En fait, le maintien à domicile des personnes âgées, les activités de loisirs, l'aménagement de l'espace urbain, la rénovation et l'entretien des maisons, des services de buanderie, de boulangerie, $d^{\prime}$ alimentation, des entreprises de recyclage constituent autant de services de proximité encore peu développés. Ils peuvent tout à la fois 
être créateurs d'emplois nouveaux et contribuer à la revitalisation économique et sociale des communautés locales. Le dégonflement des services publics n'empêchera pas les communautés locales de produire et de consommer plus de services collectifs de proximité. $C^{\prime}$ est là que réside une piste de développement prometteuse.

Les CDEC et les CDC appuyant ou créant ces initiatives peuvent ainsi jouer un rôle capital. Elles sont en continuité avec la dynamique communautaire déjà constituée. En même temps, elles introduisent des remises en question au sein de la dynamique communautaire traditionnelle par leur souci de la multiactivité, par la territorialisation de leur intervention, par leur inscription directe dans des activités économiques qui obligent à concilier utilité sociale et viabilité économique.

Ces interventions et ces expériences risquent cependant $d^{\prime}$ être confinées à demeure, limitées aux seuls terrains de l'innovation et de l'expérimentation. Mais si elles sont suffisamment soutenues par les pouvoirs publics à l'échelle nationale, ces nouvelles instances d'accompagnement pourront se développer sur l'ensemble des quartiers et des régions en difficulté du Québec ${ }^{8}$.

Les pouvoirs publics ne peuvent sous-estimer l'ampleur de la crise en dépit des reprises périodiques. La crise de l'emploi et la crise de l'État-providence ont ravagé les dispositifs de base de la socialisation au niveau des quartiers. Les intervenants sociaux sont souvent les premiers à le constater et à rechercher le renouvellement des institutions locales et la création de nouveaux outils. Les pouvoirs publics, même avec un financement moindre, peuvent être plus que de simples accompagnateurs. Ils peuvent davantage s'inscrire dans des soutiens de longue durée, bref ne pas se confiner à l'aide de démarrage.

\section{PRÉVENTION SOCIALE ET DÉVELOPPEMENT LOCAL : MISE EN PERSPECTIVE}

Depuis près de quinze ans, la situation sociale des communautés locales est caractérisée par l'appauvrissement, par l'extension de la pauvreté à de nouveaux groupes et par la réapparition de la pauvreté extrême (Laferrière, 1992). Elle est aussi caractérisée par l'alourdissement des responsabilités des services sociaux publics et communautaires. Elle inquiète et fait s'interroger de plus en plus d'élus locaux, nombre de paroisses des quartiers défavorisés, nombre d'intervenants sociaux professionnels, nombre de militants et de 
dirigeants des mouvements sociaux locaux (dirigeants de caisses populaires, de syndicats, d'organisations communautaires).

De nouvelles stratégies et de nouveaux outils d'intervention se sont donc mis peu à peu en place. Nous assistons ainsi à des déplacements de l'intervention des pouvoirs publics et à un renouvellement de stratégies d'action des communautés locales en milieu urbain pour reprendre en main l'ensemble de la "question sociale». On peut émettre l'hypothèse que même du côté des entreprises et des institutions financières de nouvelles sensibilités émergent (Dutrisac, 1994 ; Robitaille, 1995). Comme le dit pour la France Jacques Ion ce qui vaut peut-être pour le Québec -, il existe:

... Un mouvement de fond qui ne serait pas seulement de l'ordre de la nécessité économique. Les notions de contrat, de territoire, de partenariat sont loin d'être l'exclusivité du social. Elles imprègnent le discours de l'entreprise comme celui des politiques régionales et locales... (Ion, 1990: 44-45).

Un travail de longue haleine en prévention sociale et en développement local est à l'œuvre dans plusieurs communautés. L'ensemble des projets novateurs observés nous font provisoirement conclure que certains facteurs favorisent la réussite. Si l'on parle de prévention et de développement local, les intervenants interrogés et nos études traduisent d'abord deux ordres de préoccupations essentielles: 1) les indispensables liens des projets avec la communauté locale; 2) le niveau de compétence des porteurs de projets. De plus, les projets les plus prometteurs s'appuient généralement sur les ingrédients suivants: la proximité, l'exemplarité et la réciprocité. D'abord la proximité: il faut entendre par là que les projets doivent avoir une taille qui permettent aux personnes et aux communautés de $s^{\prime} y$ identifier et de se les approprier. Commencer par un petit projet auquel la communauté donne de l'expansion avec le temps paraît la plupart du temps judicieux. Vient en second lieu l'exemplarité, c'est-à-dire l'importance de définir dans un premier temps des projets-pilotes à petite échelle mais susceptibles par la suite de faire tache d'huile, susceptibles de servir de stimulants à d'autres groupes et communautés désireux d'innover en matière de prévention et de développement local. Enfin, la réciprocité nous apparaît un élément clé pour indiquer l'importance de la mise en réseau d'organisations communautaires et d'intervenants engagés dans un travail similaire non plus seulement à l'échelle locale, mais à l'échelle régionale et nationale. En effet, les organisations solidaires des populations défavorisées sont souvent affaiblies par leur isolement ou leur "localisme». Le soutien par le réseautage au plan régional et national est 
aujourd'hui plus que jamais essentiel à leur développement (Fréchette et Favreau, 1993).

L'arrivée de nouvelles générations d'intervenants sociaux (intervenants du secteur communautaire, intervenants de CLSC...) et d'intervenants économiques locaux (les caisses populaires, les entreprises de l'économie locale, les municipalités) coïncide avec une nouvelle structuration de la société. Ces intervenants, confrontés avec force à une pauvreté épousant de plus en plus les contours d'une véritable exclusion, $\mathrm{n}^{\prime}$ ont pas capitulé en se positionnant en attente d'une intervention de l'État. Ils participent plutôt à leur manière à la redéfinition de cet État social (Vaillancourt, 1994). Ils n'abandonnent pas la partie aux décideurs tentés par le néolibéralisme. C'est le cas aussi de plusieurs institutions locales, tels les CLSC et un certain nombre de caisses populaires ou d'économie qui constituent les bases arrière des initiatives de type CDEC ou CDC dans la revitalisation des communautés et des régions en difficulté. Nous avons voulu montrer que ce nouvel horizon de l'intervention sociale est attribuable en bonne partie à la percée au cours des années $80 \mathrm{~d}^{\prime}$ initiatives fortes de prévention et de développement local dans lesquelles les nouvelles générations de travailleurs sociaux sauront développer leur espace si elles décident d'occuper ce terrain. Ce qui est déjà commencé, d'autant plus que le secteur public de l'intervention sociale est de plus en plus saturé sur le plan de I'emploi.

\section{Notes}

1. Cet article s'inspire des travaux de recherche suivants : 1) une recherche-action portant sur la pauvreté urbaine subventionnée par la DRHC dans le cadre d'un Comité régional de relance de l'économie et de l'emploi (Favreau, 1993-1995); 2) une recherche subventionnée par SBESC sur des projets de prévention sociale (Chamberland, Fréchette, Lindsay et Hébert, 1993-1995); 3) une recherche en cours subventionnée par le MSSSQ sur de nouveaux modes d'entraide socio-économique et d'intervention sociale, notamment les cuisines collectives (Fréchette et Deslauriers, 1995-1996); 4) une recherche en cours subventionnée par le CRSH sur les entreprises communautaires et d'insertion sociale par le travail (Favreau, Lévesque et al.). Précisons que le présent texte se veut plutôt une synthèse de ces démarches et résultats de recherche que l'expression de simples résultats d'enquêtes.

2. Voir parmi d'autres Mayer-Renaud (1986 et 1990) et Wilkins et al. (1987).

3. La " construction institutionnelle " compartimente et décompose en groupes cibles en opérant des découpages administratifs. Cette construction cohabite avec une autre, la "Construction communautaire », qui, elle, amalgame les pauvres dans un ensemble 
socio-politique quasi indifférencié: les victimes du "système ". Mais à partir de là, cette dernière a opéré des découpages identitaires (les locataires, les assistés sociaux, les chômeurs, les femmes monoparentales...). De telle sorte que la "construction communautaire " traditionnelle en vient à privilégier le travail sur une base catégorielle, recoupant ainsi, partiellement du moins, la "construction institutionnelle". Dans les deux cas, mais pour des raisons différentes, on sous-estime la communauté première d'appartenance, le quartier, son réseau de voisinage et plus largement le moteur que peut constituer pour une population le «vivre ensemble» sur un territoire donné (Doucet et Favreau, 1991: 235-244).

4. Sur la crise de l'État-providence, voir Rosanvallon (1981 et 1995) et Castel (1995). Aux fins de cet article nous nous attardons principalement aux mutations liées à la pauvreté.

5. Mentionnons ici des études de cas ou des descriptions pertinentes en matière de prévention sociale: dans les écoles (Fréchette, 1995), sur les cuisines collectives et les groupes d'entraide économique (RCCQ, 1993 et 1994; Ninacs, 1995), sur les CJS (Favreau et Lévesque, 1993), sur les entreprises communautaires (Prud'homme, 1995), sur les entreprises d'insertion sociale (Bordeleau et Valadou, 1995).

Pour une présentation plus générale de la prévention sociale, voir le numéro de la revue Apprentissage et socialisation consacré à ce thème (1993). Pour un examen d'ensemble des travaux sur le développement local, voir le numéro de Nouvelles pratiques sociales sur "les régions" (Favreau et Klein, 1995), celui de la revue Coopératives et Développement sur "Les quartiers en crise : revitalisation et développement local en milieu urbain » (Favreau, 1995), de même que l'ouvrage sous la direction de Mercier et al. (1995), "Au cœur des changements sociaux: les communautés et leurs pouvoirs".

6. Les plus récentes publications sur l'expérience des CDC sont: pour l'Outaouais, Chagnon (NPS, 1995); dans la Montérégie, Fournier (Mercier, 1995) et Lachapelle (Coopératives et Développement, 1995); dans les Bois-Francs, Lapointe (Mercier, 1995). Sur l'expérience des CDEC, Morin (Coopératives et Développement, 1995), Favreau et Ninacs, (IFDEC,1993). Sur le travail de développement local en milieu rural, Lachapelle et Dionne/Saucier (NPS, 1995).

7. C'est aussi le potentiel de démonstration que fournit l'expérience américaine de DEC. Deux mille CDEC, dont $61 \%$ ont plus de 10 ans, distribuées sur trois générations, soit de 1960 à aujourd'hui. Voir L. Favreau sur l'expérience américaine dans J.-L. Laville (1994), L'économie solidaire: mise en perspective internationale. Paris: Desclée de Brouwer.

8. Mentionnons à cet effet que la Table nationale des CDC vient d'obtenir du Secrétariat à l'action communautaire du gouvernement du Québec le financement de base triennal de $18 \mathrm{CDC}$. Ce type de financement représente un progrès, d'autant plus que ce Secrétariat d'État financera directement la Table nationale pour qu'elle suscite la création de nouvelles CDC. 


\section{Références bibliographiques}

(1993). Les villes-centres des régions métropolitaines du Québec, octobre, 1993, document présenté au ministre Ryan par les maires des six principales villes-centres du Québec.

(1993). "La prévention sociale», numéro thématique de la revue Apprentissage et socialisation, vol.16, $\mathrm{n}^{\circ} 1-2: 9-79$. Hull: Presses Interuniversitaires / UQAH.

AutÈs, M. (1992). Travail social et pauvreté. Paris: Syros/Alternatives.

Blanchet, L., M.-C. Laurendeau, D. PAul et J.-F. SAuCier (1993). La prévention et la promotion en santé mentale. Préparer l'avenir. Boucherville: Gaëtan Morin Éditeur.

Bordeleau, D. et C. VAladou (1995). Agir pour l'insertion au Québec: initiatives d'insertion par l'économique. Montréal : IFDEC.

BOUCHARD, C. et al. (1991). Un Québec fou de ses enfants, rapport du groupe de travail pour les jeunes. Québec: MSSSQ.

CASTEL, R. (1995). Les métamorphoses de la question sociale. Paris : Fayard.

CHAGNON, L. (1995). "L'expérience de la Corporation de développement communautaire Rond Point», Nouvelles pratiques sociales, vol. 8, $\mathrm{n}^{\circ} 1: 63-80$.

Chamberland, C., N. Dallaire, S. Cameron, L. FréChette, J. Hébert et J. LINDSAY (1993). "La prévention des problèmes sociaux: réalité québécoise ", Service social, vol. 42, nº 3 : 55-81.

CONSEIL DES AFFAIRES SOCIALES (1992). Le Québec solidaire. Rapport sur le développement. Boucherville: Gaëtan Morin Éditeur/Publications du Québec.

CONSEIL DES AFFAIRES SOCIALES (1990). Agir ensemble, rapport sur le développement. Boucherville: Gaëtan Morin Éditeur.

CONSEIL DES AFFAIRES SOCIALES (1989). Deux Québec dans un. Rapport sur le développement social et démographique. Boucherville, Gaëtan Morin Éditeur.

CRÉECQ (1993). Stratégie pour le développement économique et communautaire des quartiers centraux de Québec (1993-1998). Québec: Comité pour la relance de l'économie et de l'emploi du Centre de Québec.

DUTRISAC, B. (1994). «Desjardins, partenaire du développement des économies locales", revue Desjardins, vol. 60, n 3: 7-11.

FAVREAU, L. (dir.) (1995). "Quartiers en difficulté : développement local et revitalisation», Coopératives et développement, vol. 26, $\mathrm{n}^{\circ} 2: 1-126$.

FAVREAU, L. et J.-L. KLEIN (dir.) (1995). "Les régions: intervention sociale, régionalisation, économie et territoire", numéro thématique de Nouvelles pratiques sociales, vol. 8, $\mathrm{n}^{\circ} 1: 29-120$.

FAVREAU, L. et Y. HURTUBISE (1993). CLSC et communautés locales: la contribution de l'organisation communautaire. Sainte-Foy: Presses de l'Université du Québec. 
FAVReAu, L. et B. LÉvesque (1993). "Les Coopératives jeunesse de services au Québec: des entreprises d'insertion pour des jeunes de milieux populaires", Apprentissage et socialisation, vol. 16, $\mathrm{n}^{\circ}$ 1-2: 9-20.

Favreau, L. et W. NinACS (1993). Pratiques de DEC au Québec : de l'expérimentation asociale à l'émergence d'une économie solidaire. Montréal : IFDEC.

FAVREAU, L. (1994). Relancer l'économie et l'emploi de nos quartiers par le développement économique communautaire. CRÉEOM, rapport de recherche, 150 pages.

FOURNIER, J. (1995). "La CDC de Longueuil, à la jonction du communautaire et du public », dans C. Mercier et al. (1995), Au cour des changements sociaux: les communautés et leurs pouvoirs. Sherbrooke: Université de Sherbrooke/RQIIAC, p. 79-98.

FRÉCHETTE, L. (1995). "De l'école à la communauté : amorce de changement social engageant des jeunes, des parents et des leaders locaux", dans C. Mercier et al. (1995), Au cour des changements sociaux: les communautés et leurs pouvoirs. Sherbrooke: Université de Sherbrooke/RQIIAC, p. 175-190.

FRÉCHETTE, L. (1994). "Ce que nous apprennent des interventions réussies en prévention sociale dans l'Outaouais", dans Quand les conditions de vie génèrent souffrance et stress, Actes du colloque R. Laurendeau. Hull: RRSSSO, p. 57-66.

FRÉCHETTE, L. et L. FAVREAU (1993). "Intervention psychosociale et communautaire en milieux défavorisés. Une expérience de coopération internationale au Pérou", dans Revue canadienne de service social, vol. 10, n० 1, p. 56-75.

Gaulejac. V. (1989). "Honte et pauvreté", Santé mentale au Québec, vol. $15, \mathrm{n}^{\circ} 2: 128-137$.

GUAY, J. (1984). L'intervenant professionnel face à l'aide naturelle. Chicoutimi : Gaëtan Morin Éditeur.

ION, J. (1990). Le travail social à l'épreuve du territoire. Toulouse: Privat.

JACQUIER, C. (1993). Les quartiers américains, rêve et cauchemar (le développement communautaire et la revitalisation des quartiers aux ÉtatsUnis). Paris: L'Harmattan.

JULIEN, C. (1995). "Brève radiographie d'une fracture sociale", Le Monde diplomatique, juin : 16-17.

LACHAPELLE, R. (1994). "Syndicats, organismes communautaires et développement économique local: l'expérience soreloise», Coopératives et Développement, vol. 26, $\mathrm{n}^{\circ} 2: 41-60$.

LACHAPELLE, R. (1995). "Les CADC comme espace d'économie sociale", Nouvelles pratiques sociales, vol. 8, $\mathrm{n}^{\circ} 1: 81-97$.

LAFERRIÈRE, G. (1992). "La dynamique communautaire montréalaise», Relations, octobre: 239-245.

LAPOINTE, C. (1995). "Entre le pouvoir et l'influence, la CDC des Bois-Francs", dans C. Mercier et al., Au cœur des changements sociaux: les communautés et leurs pouvoirs. Sherbrooke: Université de Sherbrooke/RQIIAC, p. 239-255. 
LAVILLE, J.-L. (1992). Les services de proximité en Europe. Paris: Syros / Alternatives.

LAVILLE, J.-L. (1994). L'économie solidaire. Paris: Desclée de Brouwer.

MAYER-RENAUD, M. (1986 et 1990). La distribution de la pauvreté et de la richesse dans les régions urbaines du Québec. Portrait de la région de Montréal. Montréal: CSSSM, 109 pages.

MERCIER, C. et al. (1995). Au cœur des changements sociaux: les communautés et leurs pouvoirs. Sherbrooke: Université de Sherbrooke/ RQIIAC.

MORIN, R. (1995), "L'expérience montréalaise des CDEC: à la croisée des chemins", Coopératives et Développement, vol. 26, n 2: 13-39.

NINACS, W. (1995). "Entraide économique, création d'entreprises et empowerment», Nouvelles pratiques sociales, vol. 8, $\mathrm{n}^{\circ} 1:$ 97-119.

PAUGAM, S. (1991). La disqualification sociale. Essai sur la nouvelle pauvreté. Paris: PUF, Coll. Sociologies.

PRUD'HOMme, P. (dir.) (1995). Le Chic Resto Pop. Montréal : Fides.

RCCQ (1993-1994). Rapport d'activités. Montréal: Regroupement des cuisines collectives du Québec.

Robichaud, J.B., L. Gagnon, C. Collin et M. Pothier (1994). Les liens entre la pauvreté et la santé mentale. De l'exclusion à l'équité. Boucherville: Gaëtan Morin Éditeur, 247 pages.

ROBITAILLE, J. (1995) «La caisse d'économie des travailleurs et travailleuses de Québec ». Entrevue avec le coordonnateur C. Guimont. Vie Ouvrière, $\mathrm{n}^{\circ} 255: 10-15$.

ROMAN, J. (1993).Ville, exclusion et citoyenneté. Paris: Éditions Esprit, Coll. Société.

Rosanvallon, P. (1981). La crise de l'État-providence. Paris: Seuil.

Rosanvallon, P. (1995). La nouvelle question sociale. Paris : Seuil.

SANICOLA, L. (1994). "L'intervention de réseaux dans le contexte du service social ", dans L. Sanicola (dir.), L'intervention de réseaux. Paris : Bayard.

Tremblay, D.-G. (1990). Économie du travail. Montréal: Éditions SaintMartin / Télé-université.

VAILLANCOURT, Y. (1994). "Éléments de problématique concernant I'arrimage entre le communautaire et le public dans le domaine de la santé et des services sociaux», Nouvelles pratiques sociales, vol. 7, $\mathrm{n}^{\circ} 2: 227$ 248.

WILKINS, R. et al. (1987). L'état de santé des Québécois : un profil par région socio-sanitaire et par département de santé communautaire. Québec: Publications du Québec. 